|| Print ISSN: 2589-7837 || Online ISSN: 2581-3935 ||

International Journal of Medical Science and Diagnosis Research (IJMSDR)

Available Online at www.ijmsdr.com

NLM (National Library of Medicine ID: 101738824)

Case Report

Volume 5, Issue 6; June:2021; Page No. 15-22

\title{
TWO DIFFERENT CASES OF STROKE IN YOUNG
}

Vedhanayagam $N^{1}$, Balasenthilkumaran $M R^{2}$, Balajisundaresan $S^{3}$, Balu $A K^{4}$, Suganya $G^{5}$

${ }^{1}$ MBBS, DNB (MEDICINE), DNB (NEUROLOGY)

${ }^{2}$ MBBS, DNB Neurosurgery Consultant Neurosurgeon, Kongunad Hospitals, Coimbatore

${ }^{3}$ MBBS, MD, DM Cardiology Consultant, Cardiologist, Kongunad Hospitals

${ }^{4}$ MBBS Resident

${ }^{5}$ MBBS MDRD Radiodiagnosis Consultant Radiologist, Kongunad Hospitals, Coimbatore

\section{Conflicts of Interest: Nil}

Corresponding author: Vedhanayagam $\mathrm{N}$

DOI: https://doi.org/10.32553/ijmsdr.v5i6.807

\section{Abstract:}

We report two different cases of stroke in young. First one is, a vertebral artery dissection following ferris wheel ride at amusement park which emphasise the clinical history in early diagnosis of stroke in young. The other one is, a recurrent stroke in marfan syndrome associated with basilar invagination and isolated atrial septal aneurysym which is rare and has unique way of management.

Keywords: Stroke in young, Vertebral artery dissection(VAD), Marfan syndrome, Atrial septal aneurysym (ASA),Basilar invagination

\section{Introduction:}

Vertebral artery dissection is an important cause of posterior circulation ishaemia in young.Even without predisposing conditions for vertebral artery dissection there is a definite temporal correlation was present between neck movement, trivial trauma and acute arterial dissection[1].

Case report 1: A 22year female, presented with sudden neck pain, vertigo and tendency to fall on left side ,three days following ferris wheel ride at amusement park. On examination patient has left appendageal ataxia with torsinal nystagmus without any evidance of long tract signs. Investigations revealed a normal haemogram, blood smear ,serum B12,homocysteine,thyroid stimulating hormone,coagulation profile, lipid profile,ECG,Echo cardiogram, APLA,ANA profile and vasculitic panel.MRI brain showed acute left cerebellar infarct (Figure1:MRI -DWI showing acute left cerebellar infarct)and MR angiogram revealed normal intracranial angiogram.

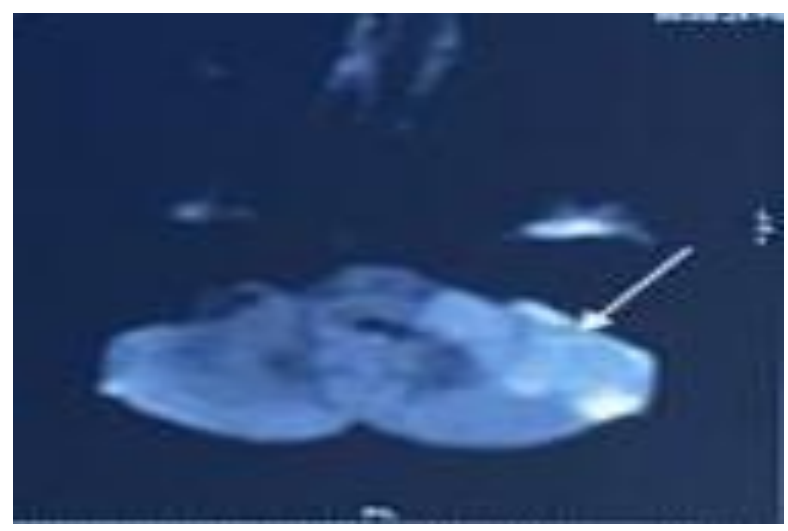

In view of neck pain, CT angiogram of neck and intracranial vessels were done which reveals V3 segment left vertebral artery dissection with significant stenosis(Figure2;Left vertebral arterial dissection with stenosis) 
The patient received intravenous heparin and aspirin for 5 days . She recovered completely without any neurological deficits.Patient discharged with oral anticoagulation afogatran $110 \mathrm{mg}$ BD and aspirin 75 mg OD for 3 months. Follow up MR angiogram showed complete resolution of dissection with no flow limiting stenosis(Figure3:Normal neck and intracranial angiogram)
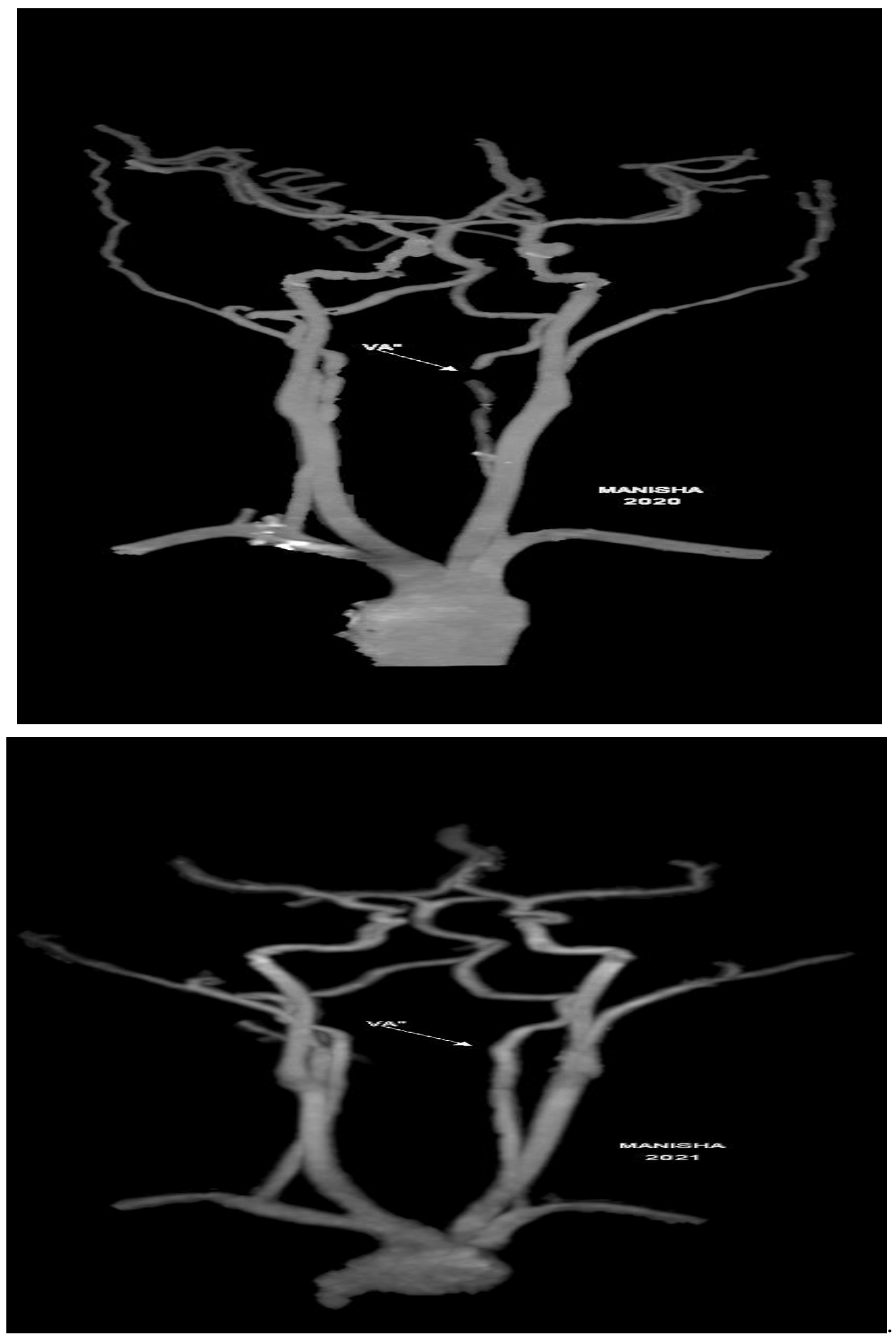
Now patient was aymptamatic and free from oral anticoagulants and aspirin.

\section{Discussion:}

Most cases of vertebral artery dissection(VAD) are in the age group of 25-55 years, with a slight female preponderance.Headache and neck pain are important warning symptoms of dissection which precedes neurological features by hours, days or rarely weeks.Pain is possibly caused by excitation of nociceptors in extracranial cerebral arteries[2] .As per review of numerous liteatures, the various predisposing conditions for VAD are heritable connective tissue disorders ( Ehlers-Danlos syndrome , Marfan's syndrome, Autosomal dominant polycystic kidney disease,Osteogenesis imperfecta type and Fibromuscular dysplasia), Hypertension , migraine, recent repiratory tract infection and hyperhomocystenaemia[3,4,5]. Other associated precipitating events are sudden neck movements during coughing, sneezing ,vomiting,practicing yoga, painting a ceiling and during chiropractic manipulation of the neck[6].Most of the traumatic dissections involve the atlanto occipital segment are due to increased mobility, poor anchoring into the neighbouring tissue, increased mechanical torsion and stretch at $\mathrm{C} 1-\mathrm{C} 2$ region[7] .

Management consists intravenous heparin followed by oral oral anticoagualtion for all patients with acute dissections of the vertebral artery, regardless of the type of symptoms, unless there are contraindications such as the presence of a large infarct with associated mass effect, hemorrhagic transformation of the infarcted area, an intracranial aneurysm, and intracranial extension of the dissection with or without SAH[8].Endovascular therapy can be reserved for cases with recurrent neurological events despite on antithrombotics and who are all not a canditate for surgical therapy.Surgical therapy consists of insitu interposition of graft or extracranial intracranial bypass only for patients with persisting symptoms refractory to maximal non invasive management and who are not candidates for endovascular therapy.Continuation of anticoagulation therapy may be guided by MRA or ultrasound.Persisting irregularity or stenosis at 3rd month necessitates continuation of therapy for another 3 months. If features of stenosis persist beyond 6 months, it is advisable to shift the patient on antiplatelet agents[8,9].

Nearly $75 \%$ of patients make excellent recovery and overall death rate is less than 5\%.Risk of spontaneous dissection approximates $2 \%$ in the first month and decreases to about $1 \%$ per year.Dissection usually does not recur in the same vascular territory[10].By conclusion we emphasise the clinical history elicitation of neck movement related trivial trauma are most important even without predisposing conditions for vertebral artery dissection in stroke management.

\section{Case report2:}

A 15 year male presented with sudden onset of left upper and lower limb weakness .On general examination patient had features of marfan syndrome (Height $157.4 \mathrm{~cm}$, arm to arm distance $175.2 \mathrm{~cm}$ height arm ratio 1.11 , arachnodactyly high arch palate ) ;Central nervous system examination showed right ataxic hemiparesis with right upper limb apraxia.Other systemic examinations were normal.Complete blood counts ,renal ,liver parameters were normal.MRI brain showed acute infarct seen in left side splenium of corpus callosum(Figure4:MRI-DWI Brain showing acute left splenial infarct) and Basilar invagination (platybasia) with tip of the odontoid process seen indenting the medulla oblongata(Figure5:MRI showing Tip of odontoid impinging on medulla)

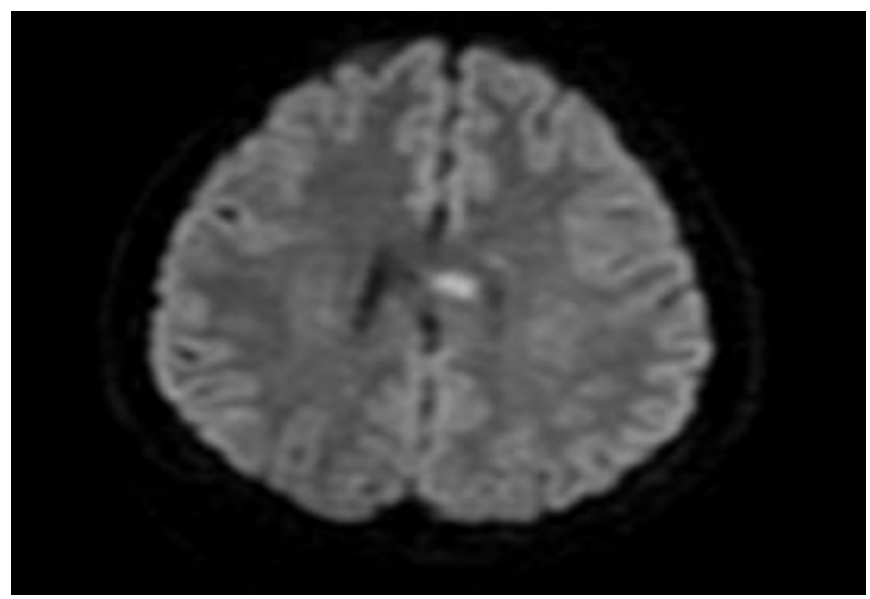




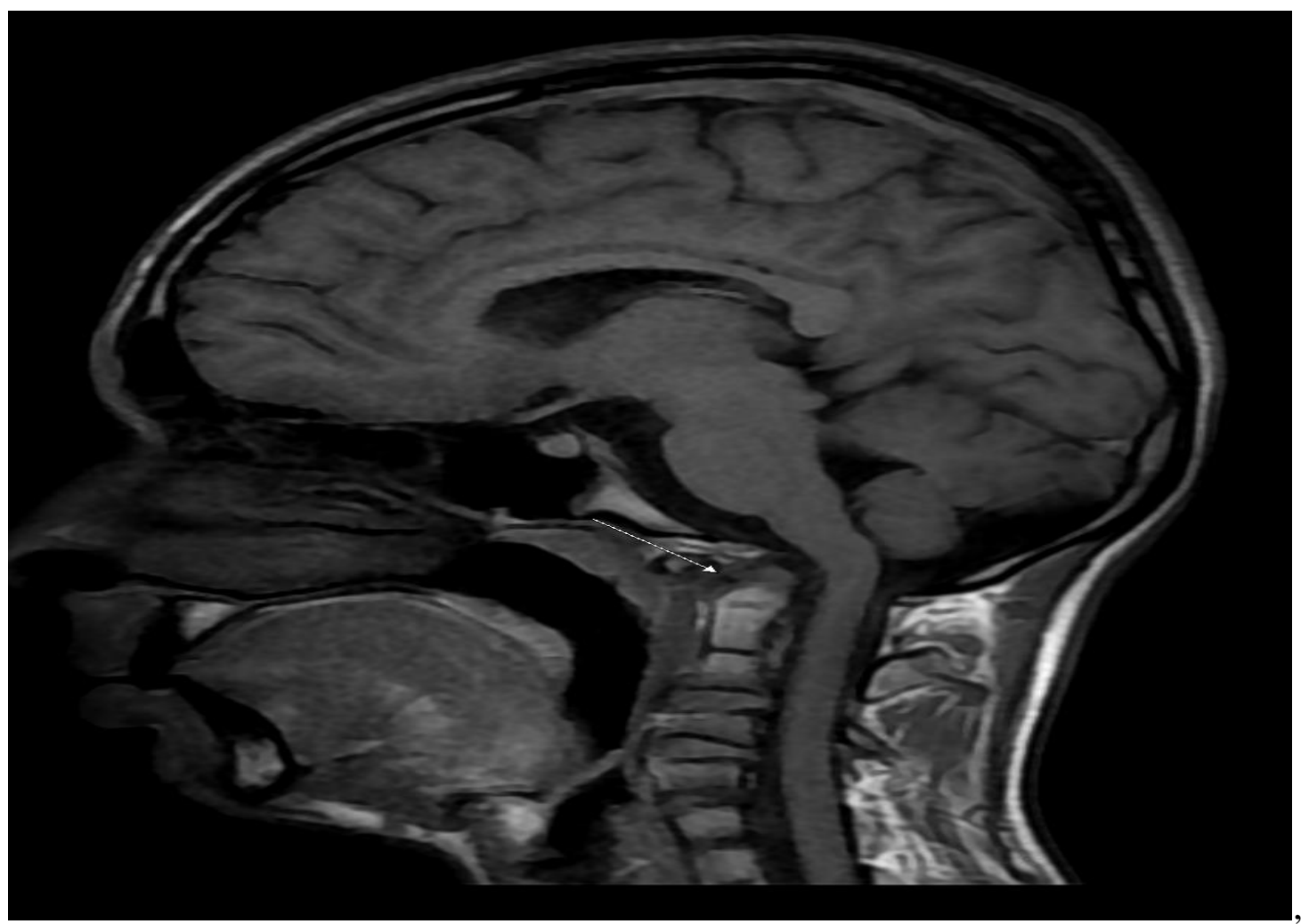

Along with cervicomedullary compromise at foramen magnum was noted.Whole spine screening revealed multiple vertebral segmentation anomaly noted as congenital block vertebrae at C2, C3, C6, C7 \&D1 levels. Transthoracic echo cardiogram showed atrial septal aneurysm with no other congenital cardiac abnormalities(Figure6:Echo showing ASA)

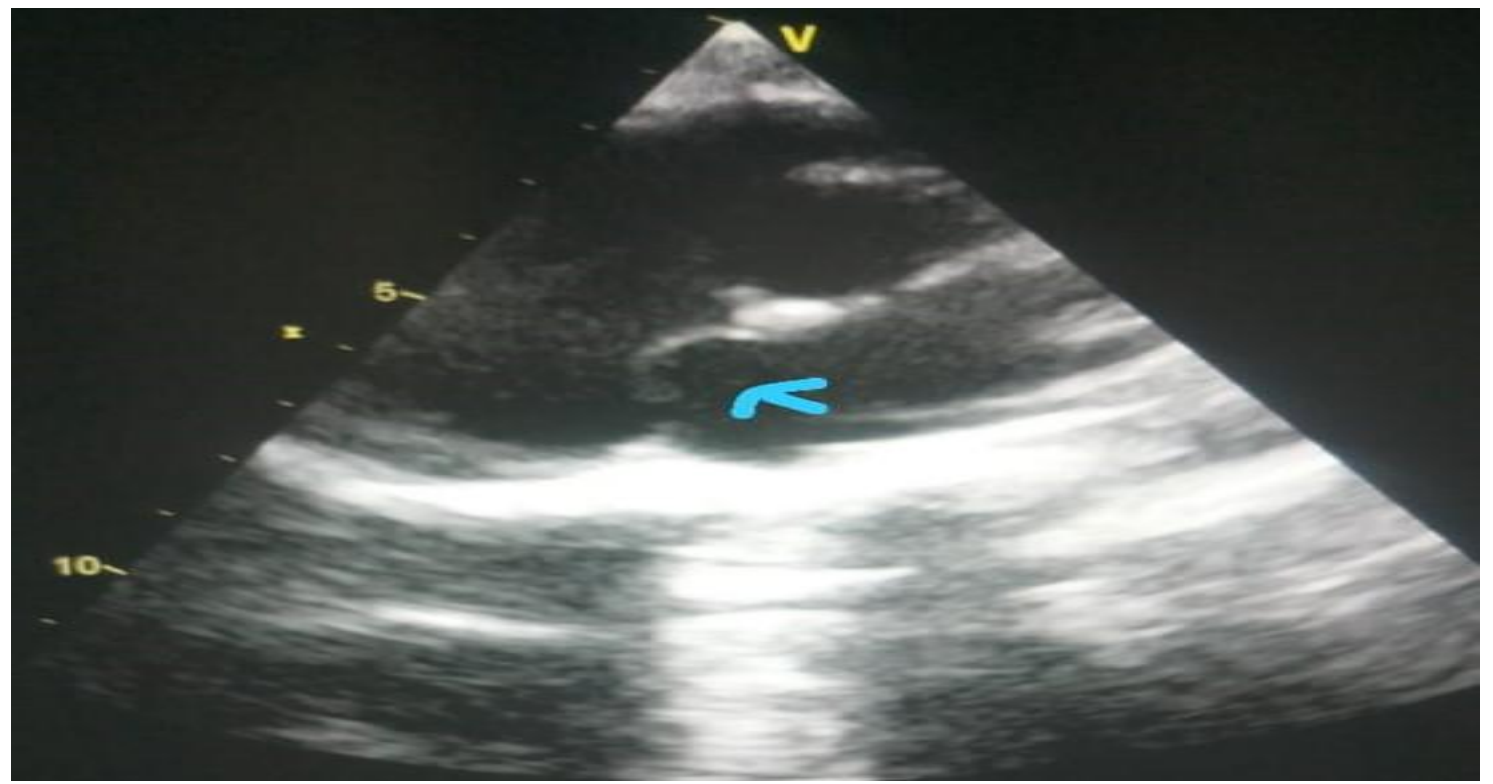

Blood investigations for young stroke profile including ANA profile, serum APLA, pANCA, cANCA, serum homocysteine, B12 were normal.Patient had improvement with low molecular heparin, dual antiplatelts(aspirin $75 \mathrm{mg}$ clopidogrel 75mg) and physiotherpy.On day 4 patient developed recurrent acute infarct in right cerebellum and in both sides of pons(Figure7:Acute right cerebellar infarct,Figure8:Acute pons infarct) 

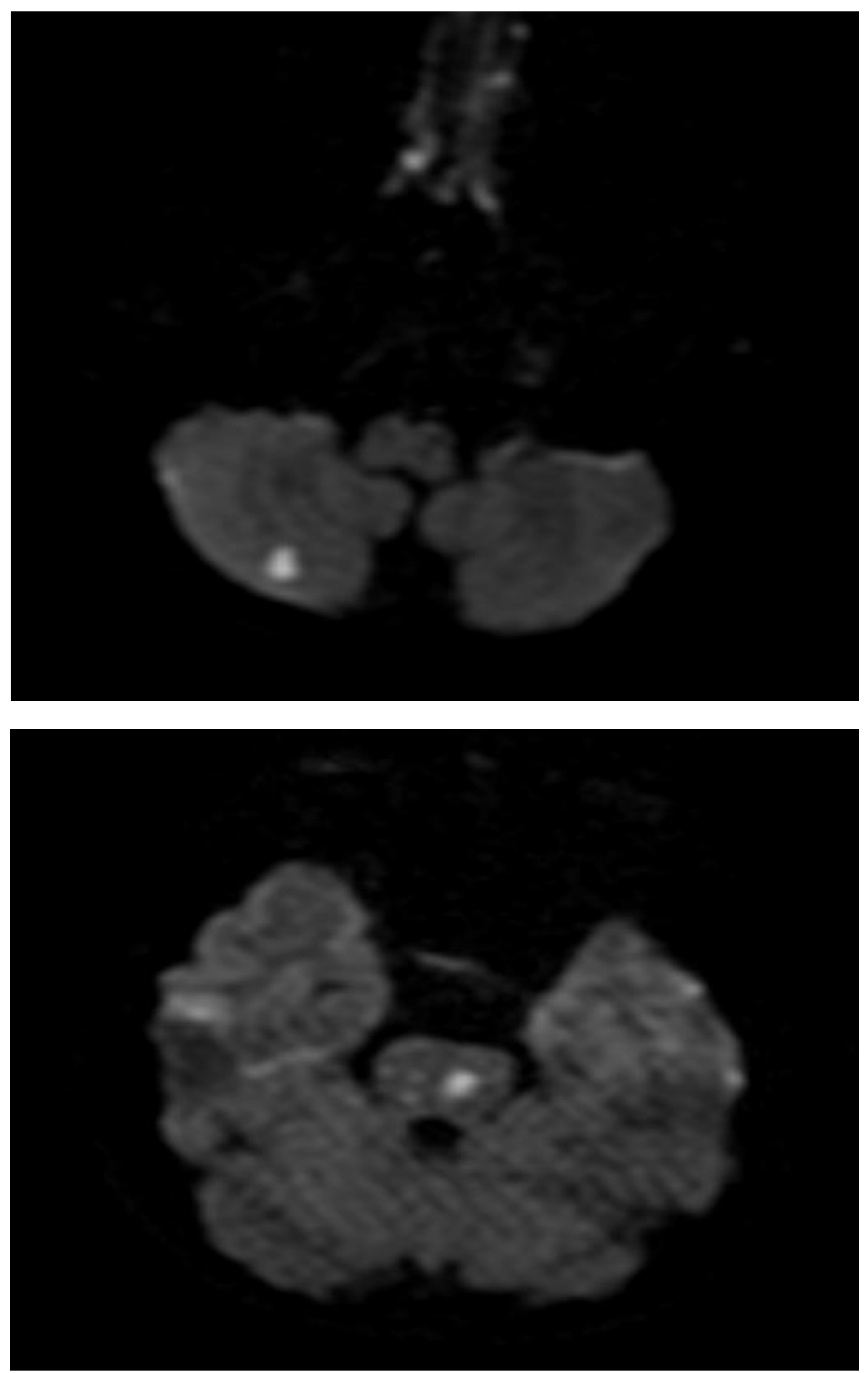
MR angiogram including neck and intracranial vessels were normal

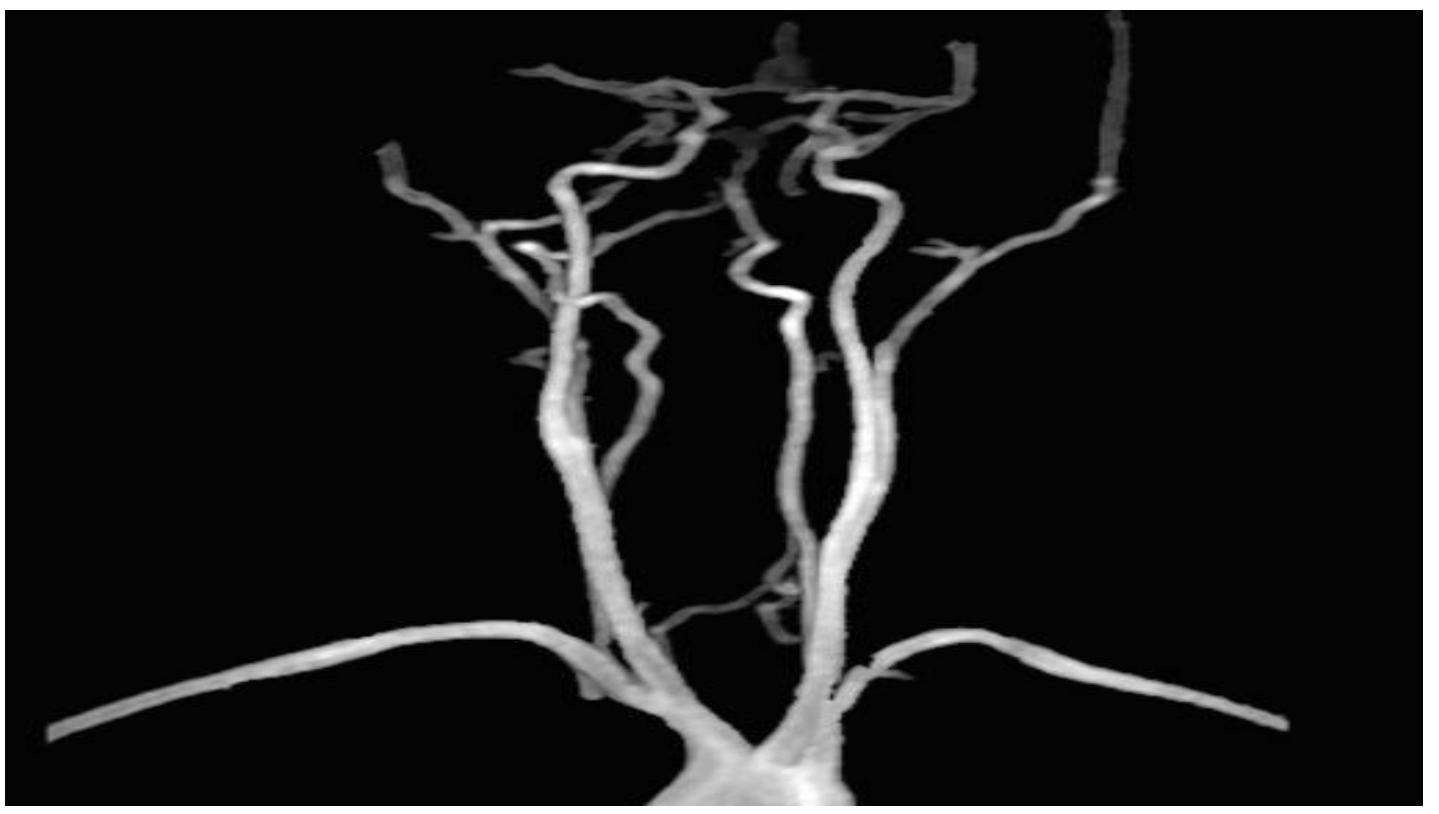

(Figure 9: MRA-showing Normal neck and intracranial angiogram) .Patient continued with the dual antiplatelets and oral anticoagulant.Patient improved significantly and became ADL(activities of daily living) independant in next 2 days.As he had platybasia with dens impinging on medulla oblongata ,procedure related neurovascular compromise was expected during transesophageal echo cardiogram ,hence cardiac CT with angiogram was done.There is an isolated atrial septal aneurysym of $1.5 \mathrm{~cm}$ width and $12 \mathrm{~cm}$ depth from inferior portion of the septum protruding into right atrium, with no thrombus or vegetation documented following cardiac CT

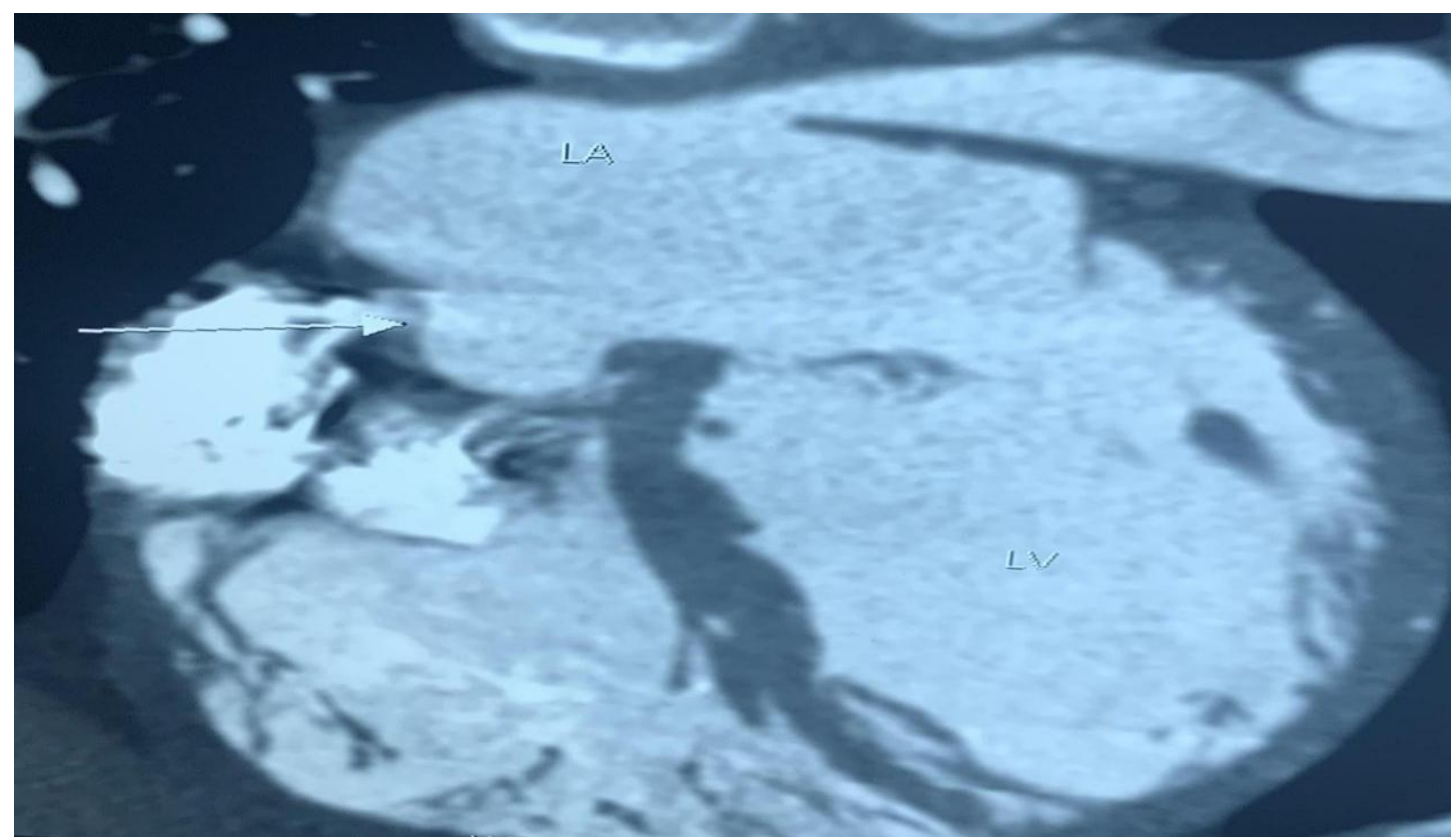

(Figure10: Cardiac CT showed atrial septal aneurysym). Holter monitoring (72 hours) did not reveal any cardiac arrhythmias. Clinical management of this case is discussed. 


\section{Introduction:}

Basilar invagination is known for dynamic flow reduction and vertebrobasilar insufficiency during neck movements(11).Isolated atrial septal aneurysym has causal association with stroke in young(12). Combination of these conditions, as in our case require guidelines for further management.

\section{Discussion:}

In our case scenario, marfan syndrome associated with platybasia and isolated atrial septal aneurysym with recurrent posterior circulation stroke will think in favour of either vertebrobasilar compromise from dens impinging on medulla oblongata or moving thrombi from isolated ASA or due to arrythmia.As per Kumar et al basilar invagination will increase the chances of vertebrobasilar stroke by direct compression on neurovascular structures(11). There are lot of ultasonagraphic studies on dyanamic flow reduction during neck movements for documenting VBI. In our case MR neck and intracranial angiogram showed normal flow during the study. As he was symptomatic with acute stroke we did not document or studied about flow reduction during neck movements.

It is well known that there is a causal relationship between stroke and atrial septal aneurysm(13). Atrial septal aneurysm may be isolated or associated patent foramen ovale (PFO), atrial septal defect, mitral valve prolapse, tricuspid valve prolapse, marfans syndrome, sinus of valsalva aneurysm and aortic dissection $(14,15)$. The embolic potential of atrial septal aneurysm has been claimed by several authors reporting systemic or pulmonary embolism by documenting the presence of a thrombus at the base of an aneurysm at autopsy and also by partly organized thrombus in histology $(16,17)$. As per Hanley et al interatrial septal aneurysm can act as an arrhythmic focus for generating atrial arrhythmias in 20 (25\%)out of 80 patients (18).But in our case it is an isloated ASA without any atrial shunts. Hence, we can able to explain the unusual nature of systemic emboli from isolated ASA as the cause for the stroke. The another point which slightly supports our thought process of ASA, probably not causing recurrent stroke is, no arrythymia demontrated by 72 hours holter monitoring, however we didn't rule out arrythmia in our case.

Uncomplicated and isolated ASA requires no specific treatment other than follow-up. Therapeutic options for prevention of recurrent strokes in patients with atrial septal aneurysm as well as atrial septal abnormality (PFO, ostium secondum ASD) are medical therapy with antiplatelet agents or anticoagulants and surgical or percutaneous closure of the defect. To prevent recurrent paradoxical embolisms in the presence of shunts, it is preferable to close the shunt either by surgical or interventions(19).

There is clear indication of life time oral anticoagulants if ASA is associated with stroke. In our case there is no documented arrhythmia or else documented posterior circulation flow reduction during neck movements.As our case had recurrent stroke we advised neurosurgical stabilization by opening of joints between C1-C2 on either side with placement of goel cage and posterior fixation and also advised to take oral anicoagulant afogatran $110 \mathrm{mg}$ twice daily until to rule out arrythmia by prolonged ambulatory cardiac monitoring(20).

\section{Conclusion:}

Platybasia with neurovascular compromise requires neurosurgical intervention to prevent stroke and we must aware of atrial septal aneurysym is known for its arrythmogenic nature and stroke in young.

\section{References:}

1. Prabhakar S, Bhatia R, Khandelwal N, Lal V, Das C P. Vertebral artery dissection due to indirect neck trauma: an under recognised entity. Neurol India 2001;49:384

2. Mokri B, Houser OW, Sandok PA et al: Spontaneous dissections of the vertebral arteries. Neurology 1988; 38: 880-885.

3. Deck JHN: Pathology of spontaneous dissection of intracranial arteries. Can J Neurol Sci1987; 14 : 88-91 
4. Grau AJ, Brandt $T$, Forsting $M$ et al: Infection-associated cervical artery dissections. Stroke1997; 28 : 453-455

5. Gallai V, Caso V, Paciaroni $\mathrm{M}$ et al: Mild hyperhomocystenemia: a possible risk factor for cervical artery dissection. Stroke2001; $32: 714-718$

6. Norris JW, Beletsky V, Nadareishvili ZG: Sudden neck movement and cervical artery dissection. The Canadian stroke consortium. CMAJ2000; 163: 38-40.

7. Weintraub MI: Beauty parlour stroke syndrome. Report of 5 cases. JAMA 1993; 269 : 2085-2086

8. Schievink WI: The treatment of spontaneous carotid and vertebral artery dissections. Curr Opin Cardiol 2000; 15 : 316-321

9. Padhi R, Kandasamy S, Kumaran B, Bheemarao PM, Kumaran S. Endovascular Management of Iatrogenic Vertebral Artery Transection During Anterior Cervical Spine Surgery: A Case Report. Neurospine. 2021;18(1):245-249. doi:10.14245/ns.2040478.239

10. 1Basseti C, Carruzzo A, Sturzenegger $\mathrm{M}$ et al : Recurrence of cervical artery dissection: a prospective study of 81 patients. Stroke1996; 27: 1804-1807.

11. Kumar M, Mehta P, Garg PK. Basilar invagination presenting as recurrent posterior circulation stroke with locked-in syndrome in a child: a case report. Int $\mathbf{J}$ Contemp Pediatr 2018;5:271-4

12. Gallet B, Malergue MC, Adams C, Saudemont JP, Collot AM, Druon MC, et al. Atrial septal aneurysm a potential cause of systemic embolism. Br Heart J. $1985 ; 53: 292-7$
13. Belkin RN, Hurwitz BJ, Kisslo J. Atrial septal aneurysm: Association with cerebrovascular and peripheral embolic events. Stroke. 1987;18:856-62.

14. Silver M. D., Dorsey J. S. Aneurysms of the septum primum in adults. Arch Pathol Lab Med. 1978; 102: 62-65.

15. Iliceto S., Papa A., Sorino M, Rizzon P. Combined atrial septal aneurysm and mitral valve prolapse: detection by twodimensional echocardiography. Am J Cardiol. 1984; 54: 1151-1154.

16. Rahko P. S., Xu Q. B. Increased prevalence of atrial septal aneurysm in mitral valve prolapse. Am J Cardiol. 1990; 66: 253-257.

17. Grosgogeat Y, Lhermitte F, Carpentier A, Facquet J, Alhomme P, Tran T. Aneurysm of the interauricular septum revealed by a cerebral embolism. Arch Mal Coeur Vaiss. 1973;66:169-77.

18. Hanley PC,Tajik AJ, Hynes JK, Edwards WD, Reeder GS, Hagler DJ, et al. Diagnosis and classification of atrial septal aneurysm by two dimensional echocardiography: Report of 80 consecutive cases.

19. Wahl A.,Krumsdorf U., Meier B., et al. Transcatheter treatment of atrial septal aneurysm associated with patent foramen ovale for prevention of recurrent paradoxical embolism in high-risk patients. J Am Coll Cardiol 2005; 45: 377-380.

20. A Kaura, L Sztriha, F K Chan, J AeronThomas, N Gall, B Piechowski-Jozwiak, J T Teo, P6542-Early Prolonged Ambulatory Cardiac monitoring in Stroke (EPACS): an open-label randomised controlled trial and economic evaluation, European Heart Journal, Volume 40, Issue Supplement_1, October 2019, ehz746.1132. 